\section{Adenosine dreams}

\author{
By Lev Osherovich, Senior Writer
}

Adenosine's role in stimulating sleep has been known since the 1980 s, but efforts to target the molecule and its receptor have been stymied by uncertainty about where the adenosine pathway acts in the brain and questions about how targeting the pathway could affect cognition.

Two separate mouse studies now show how astrocyte-derived adenosine acts on the adenosine $A_{1}$ receptor (ADORA1) to control sleep quality. The findings from one study have already led to the formation of Gliacure Inc., a start-up that hopes to modulate adenosine production by astrocytes to treat sleep disorders, epilepsy and other conditions involving intercellular communication in the brain.

The studies, published in Neuron ${ }^{1}$ and the Journal of Neuroscience $^{2}$, help elucidate the neurological mechanisms governing sleep, wakefulness and cognition, which are complex and poorly understood. ${ }^{3}$

The studies also suggest that benefits potentially could be derived by either blocking or stimulating ADORA1, depending on the desired outcome.

Philip Haydon, professor of neuroscience at Tufts University and an author on the Neuron paper, told SciBX that brain-specific adenosine pathway modulators could potentially be used to treat insomnia, shift work sleep disorder and the cognitive consequences of sleep deprivation. Haydon is a founder of Gliacure.

Many sleep drugs on the market and in clinical testing target the $\mathrm{GABA}_{\mathrm{A}}$ receptor as well as the serotonin $\left(5-\mathrm{HT}_{2 \mathrm{~A}}\right)$ receptor (HTR2A). Although GABA $_{\mathrm{A}}$ modulators like benzodiazepines can knock people out for the night, prolonged use can lead to tolerance, dependence and cognitive problems. Serotonin receptor antagonists also can cause emotional and cognitive problems.

On the wakefulness side, ADORA1 is the primary target of caffeine. However, caffeine antagonizes ADORA1s everywhere in the brain and the cardiovascular system, not just the brain regions involved in sleep, and it can cause systemic stimulation. Indeed, a range of ADORA1 antagonists are in development for cardiac indications. For example, Adentri (CVT-124; BG9928) from CV Therapeutics Inc., is in Phase III trials for congestive heart failure.

Meanwhile, Cephalon Inc.'s Provigil modafinil and Nuvigil armodafinil, which are marketed for excessive sleepiness in a variety of settings, promote wakefulness through unknown mechanisms.

\section{The adenosine scene}

Adenosine is a nucleoside that functions as a signaling molecule when outside the cell. Haydon and co-workers previously discovered that extracellular adenosine in the brain came primarily from astrocytes, a type of glial cell that modulates the activity of neurons. ${ }^{4}$ In their new study, the team examined the effect of disrupting adenosine production on sleep.

The researchers hooked up mice with a genetically engineered defect in astrocyte adenosine production to an electroencephalograph after depriving them of sleep. Ordinarily, mice deprived of sleep and then allowed to rest show high levels of slow-wave brain activity, a hallmark of deep and restful sleep. Indeed, sleep-deprived wild-type mice sleep longer and deeper than well-rested controls.

However, the adenosine mutants had less slow-wave activity than similarly sleep-deprived wild-type mice. Perhaps as a result, the mutant mice didn't need extra sleep to recover from deprivation.

"When we knock out adenosine production in the astrocytes, even sleep-deprived animals don't need to sleep more," said Haydon. "They give up sleep without paying the cost."

Blocking adenosine production also decreased the detrimental effects of sleep deprivation on long-term memory. Wild-type mice temporarily deprived of sleep failed to recognize familiar objects, whereas adenosine mutants could still distinguish between familiar and new objects.

To Haydon, this finding suggests that blocking adenosine's effects could make it possible to "stay up late and still consolidate memories."

\section{Head to bed}

In a second paper, in the Journal of Neuroscience, a team at the University of Texas Southwestern suggested that activating adenosine receptors can improve the quality of sleep without changing its duration. The study examined the effect of knocking out the receptor on slow-wave activity and short-term memory in mice.

"People with severe sleep deprivation compensate by activating the $A_{1}$ receptor and having more slow-wave sleep," said team leader Robert Greene, professor of psychiatry at UT Southwestern. However, he added that it previously was unknown how changing slow-wave activity would affect short-term memory, thus making it unclear whether ADORA1 activity should be turned up or down to treat the cognitive consequences of sleep deprivation.

The Texas team found that mice with a brain-specific deletion of the Adora1 gene had normal sleep behavior but had lower slow-wave brain activity after sleep deprivation than wild-type mice.

The team found that there was a downside to lower slow-wave activity, however: in an assay of short-term memory, sleep-deprived Adora1 knockouts performed worse than wild-type controls. 


\section{TARGETS \& MECHANISMS}

Greene said the findings show that "not all sleep is the same. Now we know that slow-wave sleep affects cognition. To have effective sleep, you need to have $\mathrm{A}_{1}$ receptor activation."

Haydon told SciBX that slow-wave activity appears to have opposite effects on short-term and long-term memory, so the studies are "highly complementary."

The Texas study shows that "slow-wave activity buildup during sleep restriction is essential for working memory cognitive performance" and thus "might point to an adaptive function" for slow-wave activity, Haydon noted.

\section{Quality over quantity}

Together, the studies suggest that blocking ADORA1 signaling could buy a few more waking hours, whereas agonizing the receptor could improve the quality of sleep.

Greene thinks transiently agonizing the receptor could increase slow-wave activity and potentially boost short-term memory after sleep deprivation. His team is designing genetic experiments to conditionally increase adenosine levels.

The good news is that ADORA1 is a G protein-coupled receptor and should be druggable with a brain-permeable agonist.

Haydon cautioned, however, that an agonist approach would run the risk of hitting the receptor elsewhere in the body, like caffeine does. Instead, he favors tinkering with adenosine production by astrocytes.

"If you want to reduce drowsiness, you would want to reduce the amount of adenosine," said Haydon. "For people with sleeping difficulties, increasing adenosine would be better."

Haydon's next step is to identify druggable targets in astrocytes, a project that he said will be taken up in part by Gliacure.

Neither group has patented their discoveries. Haydon declined to disclose Gliacure's IP position.

Greene noted that he is a consultant for sleep drug company Sepracor Inc.

Osherovich, L. SciBX 2(9); doi:10.1038/scibx.2009.347

Published online March 5, 2009

\section{REFERENCES}

1. Halassa, M.M. et al. Neuron; published online Jan. 28, 2009; doi:10.1016/j.neuron.2008.11.024

Contact: Philip G. Haydon, Tufts University School of Medicine, Boston, Mass.

e-mail: philip.haydon@tufts.edu

2. Bjorness, T.E. et al. J. Neurosci.; published online Feb. 4, 2009; doi:10.1523/JNEUROSCI.2942-08.2009

Contact: Robert W. Greene, University of Texas Southwestern, Dallas, Texas

e-mail: robertw.greene@utsouthwestern.edu

3. Mignot, E. et al. Nat. Neurosci. 5, 1071-1075 (2002)

4. Pascual, O. et al. Science 310, 113-116 (2005)

COMPANIES AND INSTITUTIONS MENTIONED

Cephalon Inc. (NASDAQ:CEPH), Frazer, Pa.

CV Therapeutics Inc. (NASDAQ:CVTX), Palo Alto, Calif.

Gliacure Inc., Boston, Mass.

Sepracor Inc. (NASDAQ:SEPR), Marlborough, Mass.

Tufts University, Boston, Mass.

University of Texas Southwestern, Dallas, Texas 